\title{
Influencia del espesor del recubrimiento de elementos de hormigón armado expuestos a procesos de corrosión y sometidos a cargas externas
}

\author{
R. S. Meneses ${ }^{1}$, J. M. Moro ${ }^{1}$, R. R. Aveldaño ${ }^{1}$, N. F. Ortega ${ }^{1}$ \\ ${ }^{1}$ Departamento de Ingeniería, Universidad Nacional del Sur, Bahía Blanca, Argentina.
}

\begin{abstract}
Información del artículo
DOI:

http://dx.doi.org/10.21041/ra.

v6i2.134

Artículo recibido el 02 de

febrero de 2016, revisado

bajo las políticas de

publicación de la Revista

ALCONPAT y aceptado el 11

de Abril de 2016. Cualquier

discusión, incluyendo la

réplica de los autores, se

publicará en el primer

número del año 2017 siempre

y cuando la información se

reciba antes del cierre del

tercer número del año 2016.
\end{abstract}

\section{(c) 2016 ALCONPAT Int.}

\section{Información Legal}

Revista ALCONPAT, Año 6, No. 2,

Mayo - Agosto 2016,, es una

publicación cuatrimestral de la

Asociación Latinoamericana de Control

de Calidad, Patología y Recuperación

de la Construcción, Internacional, A.C.

Km. 6, antigua carretera a Progreso,

Mm. 6, antigua carretera a Progra Yucatán, C.P. 97310,

Mérida Yucatán, C.P. 973
Tel.5219997385893 ,

alconpat.int@gmail.com, Página Web:

www.revistaalconpat.org

Editor responsable: Dr. Pedro Castro

Borges. Reserva de derechos al uso

exclusivo No.04-2013-011717330300-

203, eISSN 2007-6835, ambos

otorgados por el Instituto Nacional de

Derecho de Autor. Responsable de la

última actualización de este número,

última actualización de este número,

Ing. Elizabeth Sabido Maldonado, Km.

6, antigua carretera a Progreso, Mérida

Yucatán, C.P. 97310, fecha de última

modificación: 30 de mayo de 2016.

Las opiniones expresadas por los autores no necesariamente reflejan la postura del editor.

editor.

reproducción total o parcial de los

contenidos e imágenes de la publicación

sin previa autorización de la

ALCONPAT Internacional A.C.

\section{RESUMEN}

$\mathrm{Al}$ realizar estudios sobre corrosión en hormigón armado, es importante considerar en los ensayos, la acción de cargas externas, pues esta situación es la más frecuente en las estructuras. En este trabajo, se presentan los resultados obtenidos al exponer a un proceso de corrosión acelerada, a vigas de hormigón armado sometidas a esfuerzos flexionales, materializados con cargas, que generan iguales tensiones de tracción en las armaduras corroídas. Las vigas se fabricaron con diferentes espesores de recubrimiento de las armaduras y con un hormigón de resistencia característica $25 \mathrm{MPa}$. Este estudio pone en evidencia la influencia del espesor de recubrimiento de las armaduras, sobre el proceso de corrosión y su manifestación externa (fisuración del recubrimiento), frente a las mismas solicitaciones mecánicas.

Palabras claves: corrosión de armaduras traccionadas; recubrimiento; fisuración.

\section{ABSTRACT}

When studies are done in reinforced concrete, it is important to consider the action of external loads, since that is the most common situation in structures. In this study we present the results of exposing reinforced concrete beams to a process of accelerated corrosion, while under bending stresses caused by loads that generate equal tensile stresses on the corroded reinforcements. The beams were built with different cover thicknesses over the reinforcements, and with a $25 \mathrm{MPa}$ characteristic resistance concrete. This study shows the influence of the cover thickness over the corrosion process and its external manifestation (cover cracking), under the same mechanical stress.

Keywords: tension corrosion reinforcement; cover; cracking.

\section{RESUMO}

Ao realizar estudos sobre corrosão em concreto armado, é importante considerar nos ensaios a ação de cargas externas, pois esta situação é mais frequente nas estruturas. Neste artigo apresentam-se os resultados obtidos ao expor vigas de concreto armado a um processo de corrosão acelerada, submetidas a esforços de flexão materializados com cargas que geram tensões constantes de tração nas armaduras corroídas. As vigas foram elaboradas com diferentes espessuras de cobrimento das armaduras e com um concreto de resistência característica de 25MPa. Este estudo coloca em evidência a influência da espessura de cobrimento das armaduras, sobre o processo de corrosão e sua manifestação externa (fissuração do cobrimento), frente às mesmas solicitações mecânicas.

Palavras-chave: corrosão de armaduras tracionadas; cobrimento; fissuração.

Autor de contacto: Romina S. Meneses (romina.meneses@uns.edu.ar) 
Revista ALCONPAT, Volumen 6, Número 2, Mayo - Agosto 2016, Páginas 129 - 144

\section{INTRODUCCIÓN}

El hormigón es el material más empleado en la construcción, en el mundo. Las estructuras realizadas con este material, poseen una cierta vida útil, donde los procesos que provocan su degradación, afectan a la estética y, principalmente, a sus prestaciones funcionales, poniéndose en evidencia con la aparición de manchas, fisuras, desprendimientos del recubrimiento, etc.

Una de las patologías que se presentan, más habituales, en estructuras de hormigón armado o pretensado, es la corrosión de sus armaduras metálicas. El acero de las armaduras está protegido de la corrosión por una capa pasiva de productos de hidratación del cemento, conformando un ambiente alcalino. Sin embargo en ambientes marinos, los iones cloruro se acumulan en la superficie del hormigón y penetran lentamente hacia su interior, hasta llegar a las armaduras metálicas, tal que, a partir de cierta concentración disuelven la capa que pasiva al acero y se activa un proceso de corrosión. Estos productos de corrosión poseen un volumen muy superior al acero del cual se originan; primeramente, se alojan en la estructura de poros del hormigón y una vez que la misma se colmata, comienzan a producirse tensiones internas, (Peralta, 2009) que terminan generando fisuras en el recubrimiento (Alonso, 1996; Andrade, 1993).

Estas fisuras del recubrimiento favorecen el avance del deterioro de las estructuras afectadas, debido a que permiten el ingreso de agentes externos (cloruros, agua y oxígeno) hacia las armaduras, provocando una disminución de la sección del acero y afectando a la adherencia entre ambos materiales (Al-Sulaimani, 1990; Almusallam, 1996; Rodríguez, 1993). De esta manera, a medida que avanza el deterioro, disminuye el momento resistente del elemento estructural afectado, teniendo consecuencias negativas en la capacidad portante y en la vida útil de la estructura afectada(Melchers, 2001; Rodríguez, 1996; Rodríguez, 1998; Torres-Acosta, 2007).

Existen una serie de factores que controlan el proceso de fisuración del recubrimiento (Alonso, 1998), entre ellos, se destacan: el ambiente al que está expuesta la estructura (Aveldaño, 2011) y las propiedades del hormigón, desde el punto de vista de la resistencia mecánica del recubrimiento (Rodríguez, 1998), y también por la necesidad de poseer una estructura de poros que brinde estanqueidad; por ello, dentro de las propiedades del hormigón, la absorción capilar (relacionada con la estructura de poros) juega un papel muy importante, lo que ha llevado a que diversos países dicten normas que fijan valores máximos para ella (Instituto Argentino de Normalización y Certificación, 2005). En trabajos anteriores, realizados en el laboratorio donde se materializaron estas investigaciones (Schierloh, 2001; Schierloh, 2003), con vigas elaboradas con distintos hormigones y libres de cargas externas, se estableció que la absorción capilar influye en la aparición de las primeras fisuras por corrosión y la evolución de los potenciales de corrosión, durante el tiempo de los ensayos de corrosión acelerada, no hallándose correlaciones significativas, entre el cuadro de fisuración producido por la corrosión, con el comportamiento electroquímico registrado durante los ensayos. Otros factores a considerar en el proceso corrosivo y sus efectos, están relacionados con la forma en que está distribuida la armada de la estructura (Aveldaño, 2009; Hariche, 2012) y las solicitaciones mecánicas a que están sujetas las armaduras en proceso de corrosión que, inevitablemente, están asociados con el estado tensional del hormigón que las circunda (Aveldaño, 2013).

Con relación al estado tensional de las armaduras se puede decir que introduce una cierta incertidumbre en los procesos corrosivos y sus consecuencias. Pese a la importancia del tema, que en el caso de las armaduras sometidas a esfuerzos de tracción, se puede llegar a originar el fenómeno conocido como "corrosión bajo tensión", pero son escasas las investigaciones en estructuras de hormigón. No obstante, en los últimos años han aparecido algunos trabajos (Calabrese, 2013; Elfergani, 2012; Fumin, 2011; Ortega, 2011). Pese a que las estructuras de hormigón armado, en servicio, normalmente se corroen bajo cargas estáticas y/o dinámicas (por ejemplo, en puentes), sin 
Revista ALCONPAT, Volumen 6, Número 2, Mayo - Agosto 2016, Páginas 129 - 144

embargo se han realizado pocas investigaciones, en comparación con las que analizan a las estructuras en proceso de corrosión, en ausencia de cargas externas. El comportamiento que se espera de una estructura que está sometida a los efectos de la corrosión y en simultáneo bajo la acción de cargas externas (tanto estáticas, como dinámicas), es distinta la evolución de la corrosión que sino están actuando estas cargas, por estos motivos, algunas investigaciones fueron destinadas al estudio de esta temática; (Hariche et al., 2012), estudiaron vigas de hormigón armado expuestas a corrosión acelerada, donde los principales parámetros que variaron en sus investigaciones fueron la disposición de las armaduras y la magnitud de las cargas externas (Hariche et al., 2012; Yu et al., 2015), analizaron la evolución de la corrosión y la fisuración en vigas fisuradas bajo la acción constante de cargas externas y expuestas en cámara salina, realizando dos conjuntos de vigas con distintas direcciones de colada, en relación a la armadura de tracción. (Linwen, 2015 y Malumbela et al, 2009), estudiaron vigas bajo corrosión acelerada con cargas aplicadas muy inferiores a las de rotura, donde se monitoreaba la variación de la profundidad del eje neutro, la curvatura y el momento de inercia de ellas.

En este trabajo se presentan los resultados de ensayos realizados en vigas de hormigón armado con diferentes espesores de recubrimiento, sometidas a un proceso de corrosión acelerada y bajo la acción de cargas externas estáticas. Dichos ensayos tienen como objeto evaluar el comportamiento de las mismas, desde el punto de vista de la corrosión de sus armaduras y sus efectos externos (fisuración del recubrimiento del hormigón) e internos (potenciales de corrosión). En esta temática se ha trabajado mucho en vigas sin cargas externas, no habiéndose encontrado trabajos análogos al presentado en esta publicación.

\section{PROCEDIMIENTO EXPERIMENTAL}

\subsection{Materiales utilizados}

Los materiales utilizados en las mezclas del hormigón fueron los siguientes:

- Cemento: se usó Cemento Pórtland Normal (CPN40) proveniente de una fábrica ubicada en la Provincia de Buenos Aires (zona central de Argentina).

- Agregado fino: constituido por arena natural silícea procedente de un yacimiento ubicado al sur de la Provincia de Buenos Aires, que cumple con las especificaciones de calidad dadas por la Norma IRAM 1627. (Instituto Argentino de Normalización y Certificación, 1997)

- Agregados gruesos: constituido por canto rodado cuyo Tamaño Máximo Nominal era 25 mm, que cumple con los límites granulométricos indicados en la Norma IRAM 1627. (Instituto Argentino de Normalización y Certificación, 1997)

El hormigón fue elaborado con una relación agua/cemento (a/c) de 0,50, valor adoptado por la normativa argentina (Instituto Argentino de Normalización y Certificación, 2005), para estructuras resistentes que se encuentren a más de $1 \mathrm{Km}$ de la costa, en zona influenciada por vientos con sales marinas. Un detalle de la dosificación usada se muestra en la Tabla 1.

Tabla 1. Composición y características del hormigón

\begin{tabular}{|l|c|}
\hline \multicolumn{1}{|c|}{ Materiales constitutivos } & Kg/m $\mathbf{3}$ de hormigón \\
\hline Cemento Portland Normal & 350 \\
\hline Agregado fino (arena natural silícea) & 895 \\
\hline Agregado grueso (canto rodado) & 920 \\
\hline Agua potable de red & 190 \\
\hline Relación agua/cemento & 0,50 \\
\hline
\end{tabular}

Influencia del espesor del recubrimiento de elementos de hormigón armado expuestos a... 
Revista ALCONPAT, Volumen 6, Número 2, Mayo - Agosto 2016, Páginas 129 - 144

\subsection{Elaboración de muestras}

Se fabricaron 4 vigas de hormigón armado en las que se varió la relación recubrimiento / diámetro de las barras, para simular distintos grados de protección de las armaduras, y se moldearon probetas cilíndricas, con el objeto de caracterizar su comportamiento mecánico (compresión y tracción) y físico (ensayo de absorción capilar).

Las vigas poseían una sección de 8 x $16 \mathrm{~cm}$ y $220 \mathrm{~cm}$ de longitud, la armadas longitudinales eran barras de acero corrugado de dureza natural (ADN 420, IRAM-IAS U 500-528) (Instituto Argentino de Normalización y Certificación, 1989), que es el acero usado en la construcción en Argentina, con un diámetro de 4,2 mm (dos barras en la cara superior y dos en la inferior), y estribos cerrados de acero liso de diámetro: $2,1 \mathrm{~mm}$, separados cada $10 \mathrm{~cm}$, con un recubrimiento que varió entre $10 \mathrm{~mm}$ y $25 \mathrm{~mm}$. Debe aclararse, que la elección de los diámetros usados en el ensayos, se debió a la intención de mantener la semejanza geométrica de las vigas de laboratorio (en el orden de 1/3 del tamaño de la estructura real), con vigas empleadas en construcciones reales, para lo cual, se usaron barras comercialmente disponibles en este país. En la Tabla 2 se presentan las características de las armaduras.

Tabla 2. Características de las armaduras

\begin{tabular}{|l|c|c|c|c|}
\cline { 2 - 5 } \multicolumn{1}{c|}{} & $\begin{array}{c}\text { Tipo de } \\
\text { acero }\end{array}$ & $\begin{array}{c}\text { Diámetro } \\
\mathbf{( m m )}\end{array}$ & $\begin{array}{c}\text { Límite Elástico } \\
\mathbf{( M P a )}\end{array}$ & $\begin{array}{c}\text { Tensión de } \\
\text { Rotura (MPa) }\end{array}$ \\
\hline Armadura Longitudinal & Dureza Natural & $4 \times 4,2$ & 680 & 690 \\
\hline Estribos & Liso & 2,1 & 640 & 640 \\
\hline
\end{tabular}

El hormigón fue colado en los encofrados y vibrado mecánicamente, humectándolo durante los primeros 7 días. Posteriormente, el fraguado continuó en ambiente de laboratorio (temperatura $\approx$ $20^{\circ} \mathrm{C}$, humedad relativa $\approx 50 \%$ ) por aproximadamente 60 días. También se elaboraron probetas cilíndricas de hormigón (15 x $30 \mathrm{~cm}$ ), empleadas en los ensayos físicos de capacidad y velocidad de succión capilar (IRAM 1871) (Instituto Argentino de Normalización y Certificación, 2005) y mecánicos: resistencia a compresión (IRAM 1546) (Instituto Argentino de Normalización y Certificación, 1992) y a tracción indirecta (IRAM 1658) (Instituto Argentino de Normalización y Certificación, 1992). Se moldearon y curaron de acuerdo con la norma IRAM 1534 (Instituto Argentino de Normalización y Certificación, 1985).

\subsection{Proceso de corrosión acelerada}

A los efectos de solicitar con esfuerzos de tracción, a las armaduras superiores (que serían las que posteriormente iban a ser sometidas a un proceso de corrosión acelerada), se colocaron cargas en los extremos de las vigas, siendo adoptada como área a corroer una zona intermedia, dejando voladizos en los dos laterales. En la Figura 1, se aprecia el esquema estructural adoptado. La zona comprendida entre los apoyos, posee una solicitación a flexión casi constante, por ello, toda la zona de las barras afectadas por la corrosión, está sometida a una tensión prácticamente de igual intensidad, al estar sometidas en cada caso, a su peso propio, más la acción de distintas cargas que variaron desde $90 \mathrm{~kg}$ a $100 \mathrm{~kg}$. 


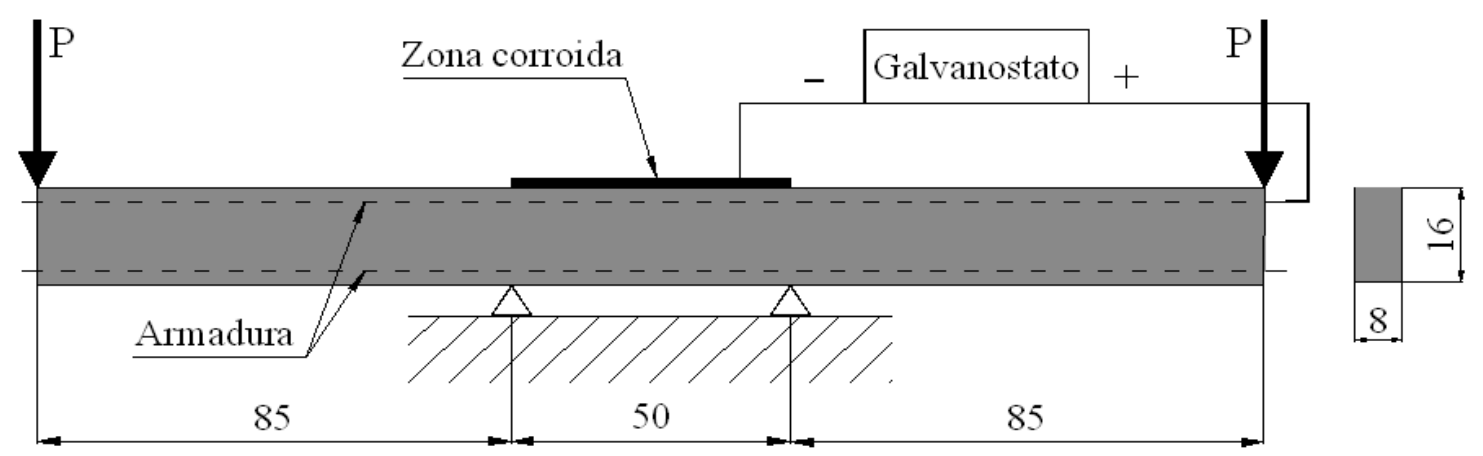

Figura 1. Esquema del ensayo

Los elementos fueron sometidos a corrosión acelerada por aproximadamente 160 días, mediante la aplicación de una corriente impresa externa, provista por un galvanostato, conectado entre un extremo expuesto de armadura superior (ánodo) y un contraelectrodo (cátodo) formado por una malla de acero inoxidable (de $50 \mathrm{~cm}$ de largo e igual ancho que la viga), ubicado en su cara superior. Sobre el mismo se dispuso una esponja (de iguales dimensiones), que se mantuvo humectada con una cantidad controlada de una solución acuosa con 0,3 \% (en peso) de cloruro de sodio ( $\mathrm{NaCl}$ ) (3 g NaCl / litro agua). Previo a conectar el galvanostato, durante 2 días se inició la humectación de la zona central, a los efectos de asegurar que todo el recubrimiento tenga la humedad necesaria para permitir la circulación de la corriente. Con el fin de tener una humectación controlada (reduciendo la evaporación), se las cubrió con una placa de acrílico y luego una cobertura de nylon. La densidad de corriente aplicada fue de $100 \mu \mathrm{A} / \mathrm{cm}^{2}$, que es alrededor de diez veces la medida en estructuras de hormigón armado altamente corroídas (Rodríguez, 1993), fue elegida por haberse adoptado en investigaciones de distintos grupos que trabajan en el tema (Acosta, 1998; Alonso, 1994; Alonso, 1998; Aveldaño, 2011), con el fin de que al finalizar el proceso en estudio se obtengan penetraciones de la corrosión superiores a 0,30 mm en las barras, en un tiempo prudencial.

Es interesante aclarar que con este método empleado para lograr una corrosión acelerada, el proceso de corrosión se inicia simultáneamente en todas las vigas al conectarlas al galvanostato, independientemente del recubrimiento que tengan. Por ello, lo que se está estudiando, en este trabajo, es el proceso de corrosión y los síntomas que genera el mismo a partir de la aplicación de la corriente exterior. Lógicamente, en estructuras existentes, afectadas por un ambiente con cloruros, las armaduras que tienen un mayor recubrimiento, tardan más tiempo en despasivarse (inicio de la corrosión) y este proceso que antecede a la corrosión es estudiado por investigadores que trabajan en penetración de cloruros y en carbonatación, líneas de investigación distintas a la de este trabajo. En el análisis presentado en este trabajo, se trata de representar el período posterior a la iniciación (denominado período de propagación de la corrosión). Los dos períodos mencionados, son los estudiados y definidos por Tuutti (Tuutti, 1982), en su conocido diagrama de vida útil de las estructuras de hormigón armado en función de la corrosión de sus armaduras.

Se supone que las armaduras afectadas por la corrosión, poseen una superficie de $162 \mathrm{~cm}^{2}$ (determinada por los $50 \mathrm{~cm}$ centrales de las barras y por los 6 estribos que están ubicados en esta zona, suponiendo que la corrosión se extendiese hasta una longitud en la rama vertical de los estribos de $1 \mathrm{~cm}$ ). Para suministrar una densidad de corriente de $100 \mu \mathrm{A} / \mathrm{cm}^{2}$, los galvanostatos proveyeron una intensidad de corriente de 16,2 mA. Al finalizar el ensayo se descubrieron las armaduras, observándose un ataque no uniforme, con presencia de picaduras (lo que indica que los cloruros actuaron sobre la superficie de las barras) y se estableció que en la armadura longitudinal, la corrosión había sobrepasado los $50 \mathrm{~cm}$ centrales, en un par de centímetros a cada lado de esta zona 
Revista ALCONPAT, Volumen 6, Número 2, Mayo - Agosto 2016, Páginas 129 - 144

(hasta donde llegó la humedad en el recubrimiento), con una profundidad de corrosión muy pequeña (se veía claramente el corrugado de las barras) y, con menor extensión, algo similar ocurrió con los estribos

En la Tabla 3 se identifican las vigas ensayadas, de acuerdo a la relación espesor del recubrimiento / diámetro de las armaduras adoptado en cada viga $(\mathrm{r} / \phi)$ y la carga aplicada $(\mathrm{P})$, cuya magnitud se varió de forma tal de producir la misma tensión en las armaduras traccionadas en todos los casos. Los análisis se realizaron empleando esta relación, como ocurre en mucha de la bibliografía del tema y no con el recubrimiento solo, a los efectos de generalizar los resultados obtenidos. Por otra parte, es interesante recordar que independientemente del recubrimiento empleado, mientras se mantenga una misma relación $\mathrm{r} / \phi$, el proceso de fisuración será prácticamente idéntico. En (Peralta M.H. et al., 2006) puede verse un estudio numérico donde se establece la evolución de las tensiones en distintos lugares del recubrimiento de hormigón, previo a la fisuración, determinándose la pertinencia de realizar el análisis empleando esta relación.

Tabla 3. Denominación de las vigas

\begin{tabular}{|c|c|c|}
\hline Denominación & $\begin{array}{c}\text { Relación } \\
\text { recubrimiento/diámetro } \\
(\mathbf{r} / \boldsymbol{\phi})\end{array}$ & $\begin{array}{c}\text { Carga aplicada (P) } \\
\mathbf{( K g )}\end{array}$ \\
\hline V90 & 6,0 & 90 \\
\hline V93 & 4,8 & 93 \\
\hline V96 & 3,6 & 96 \\
\hline V100 & 2,4 & 100 \\
\hline
\end{tabular}

\subsection{Determinaciones realizadas durante los ensayos}

Primeramente se cargaron las vigas y previo al inicio de la humectación, se midió la fisuración inicial por flexión. El seguimiento de la superficie de las vigas, se realizó observándolas diariamente y registrando el momento de aparición de las primeras manchas y de las primeras fisuras por corrosión. A partir del surgimiento de estas primeras fisuras, se efectuó periódicamente la medición del ancho (por comparación con una reglilla graduada, con una división mínima de 0,05 mm (ver Figura 2) y del largo de las mismas (con una regla graduada, que posee una precisión del milímetro). Esta tarea se efectuó para hallar los Anchos Máximos de Fisura y las Áreas de Fisuración, que se obtienen como la sumatoria de los largos de cada fisura, multiplicado por su respectivo ancho.

Paralelamente, se registraron los Potenciales de Corrosión, con el fin de efectuar un seguimiento electroquímico del proceso de corrosión (Figura 3), de acuerdo a un procedimiento normalizado (American Society for Testing and Materials, 1980). Éstos se midieron con un voltímetro marca CANIN (PROCEQ), empleando un electrodo de referencia de Cobre - Sulfato de Cobre (CSC), especialmente construido para realizar mediciones en estructuras de hormigón. Los Potenciales de Corrosión en los $50 \mathrm{~cm}$ centrales de la zona central afectada por corrosión, se determinaron en 5 puntos equidistantes promediándose el valor de los mismos. 
Revista ALCONPAT, Volumen 6, Número 2, Mayo - Agosto 2016, Páginas 129 - 144

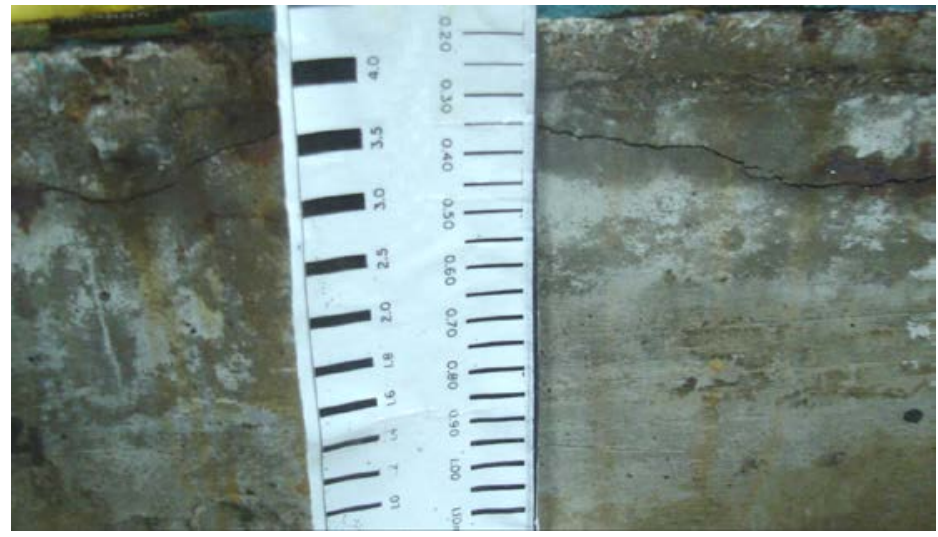

Figura 2. Medición del ancho de una fisura generada por corrosión acelerada.

Antes de comenzar las mediciones, se humectó durante 2 días, hasta alcanzar una humectación constante, con la mencionada solución de $\mathrm{NaCl}$ al 0,3 \% en peso, y posteriormente, se aplicó galvanostaticamente una densidad de corriente constante durante todo el ensayo. Bajo estas condiciones, se siguió durante todo el ensayo, la evolución del Potencial de Corrosión en diversos puntos característicos de la viga (sobre las zonas seca y húmeda, en coincidencia o no con estribos). Todas las mediciones se realizaron desconectando previamente el galvanostato, y se humedecía el hormigón en las zonas donde se realizarían posteriormente las determinaciones, de tal manera que las mediciones de los potenciales se estabilicen rápidamente. Este proceso de humectación, habitualmente, duró un par de horas.

La penetración teórica del ataque a las barras se determinó a partir de la Ley de Faraday (Alonso, 1998), suponiendo una corrosión uniforme de las armaduras, por ello, se puede decir que la penetración determinada es un valor medio, y puede estimarse mediante la expresión:

$$
\mathrm{x}=0,0116 \mathrm{I}_{\text {corr }} \mathrm{t}
$$

Dónde:

$\mathrm{x}$ : profundidad del ataque [mm];

0,0116: factor de conversión de unidades;

$\mathrm{I}_{\text {corr: }}$ : densidad de corriente de corrosión $\left[\mu \mathrm{A} / \mathrm{cm}^{2}\right]$

t: duración del ataque [años].

Esta profundidad de ataque medio en las barras, se obtiene a través de la velocidad de corrosión, que está representada por la $\mathrm{I}_{\text {corr, }}$ que es mantenida constante por el galvanostato.

\section{RESULTADOS Y DISCUSIÓN}

\subsection{Caracterización}

En la Tabla 4 se puede apreciar un detalle de los resultados obtenidos en los ensayos de caracterización de las propiedades del hormigón. 
Revista ALCONPAT, Volumen 6, Número 2, Mayo - Agosto 2016, Páginas 129 - 144

Tabla 4. Características del hormigón empleado en las vigas ensayadas

\begin{tabular}{|c|c|c|c|}
\hline $\begin{array}{l}\text { Compresión } \\
\text { (MPa) }\end{array}$ & $\begin{array}{l}\text { Tracción } \\
\text { Indirecta } \\
\text { (MPa) }\end{array}$ & $\begin{array}{c}\text { Capacidad de } \\
\text { Succión Capilar } \\
\left(\mathbf{g} / \mathbf{m}^{2}\right)\end{array}$ & $\begin{array}{c}\text { Velocidad de } \\
\text { Succión Capilar } \\
\left(\mathrm{g} / \mathrm{m}^{2} . \mathrm{s}^{1 / 2}\right)\end{array}$ \\
\hline 27,0 & 2,5 & 2.773 & 3,45 \\
\hline
\end{tabular}

De acuerdo a los resultados obtenidos experimentalmente, la velocidad de succión capilar, es inferior a $4 \mathrm{~g} / \mathrm{m}^{2} . \mathrm{s}^{1 / 2}$, valor máximo de velocidad de succión capilar permitido por la normativa argentina (Instituto Argentino de Normalización y Certificación, 2005), por ello, el hormigón resulta adecuado para estructuras ubicadas en ambientes con presencia de cloruros.

\subsection{Seguimiento electroquímico}

En la Figura 3 se muestra el comportamiento electroquímico de las armaduras, donde se graficó la evolución de los Potenciales de Corrosión, representándose el promedio de las mediciones obtenidas en 5 puntos ubicados en la zona central afectada por corrosión, en cada una de las 4 vigas ensayadas. En el eje de las abscisas hay dos escalas, una representada por el tiempo trascurrido desde que comenzó el ensayo y en la parte superior de esta figura, se colocó la Penetración Teórica del ataque, que es un valor medio fijado con la Ley de Faraday (Ecuación (1)), aplicado a las pérdidas de material en las armaduras ubicadas en la zona central de las vigas.

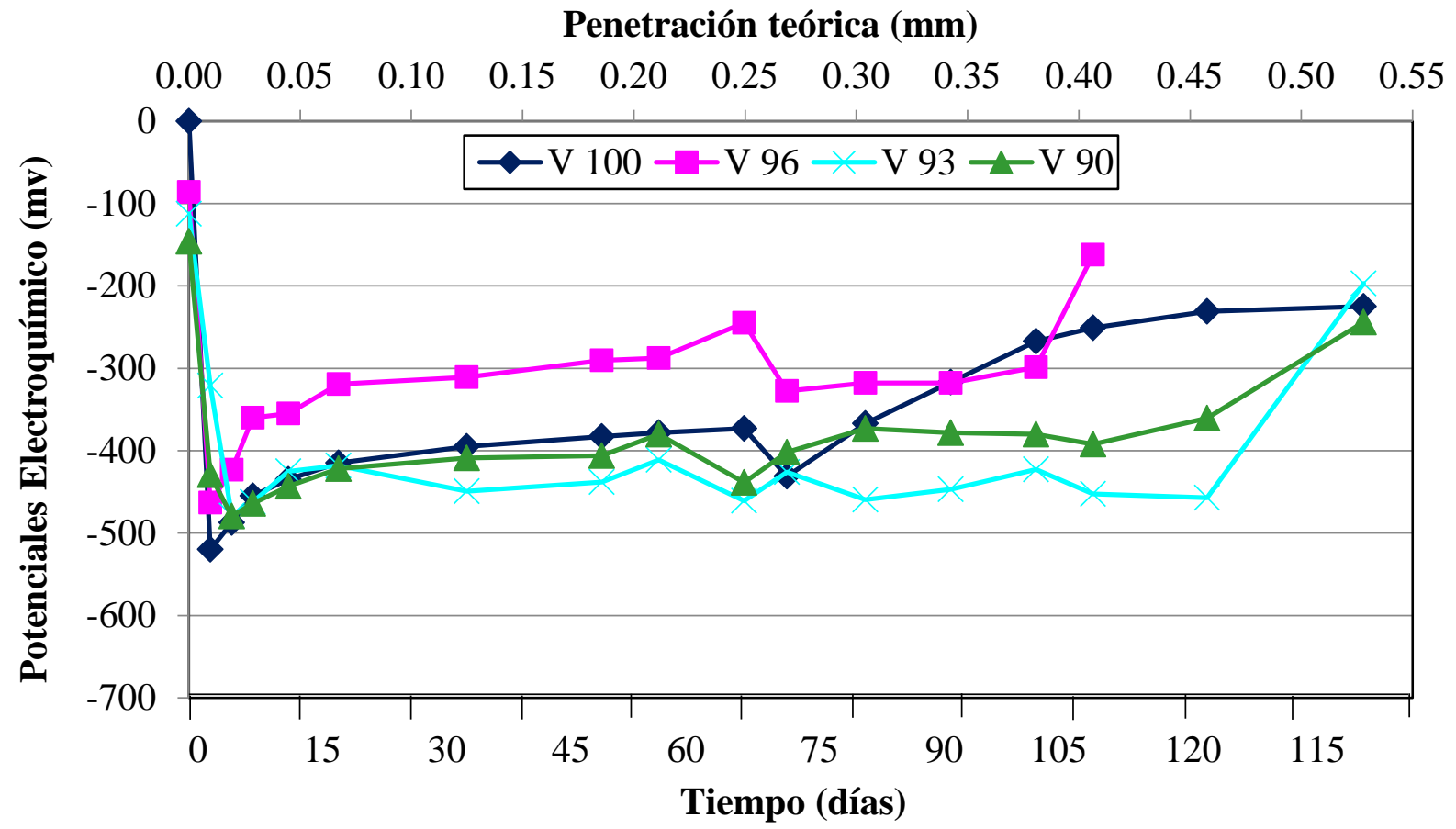

Figura 3. Potenciales de corrosión promedio (zona humectada), en función de la Penetración Teórica y del Tiempo

Cabe acotar, que si bien el ensayo se prolongó por aproximadamente 160 días, al arribar a los 140, la viga V96 llegó al colapso (Figura 4), debido a una importante reducción de la sección transversal de su armadura, producida por la profundidad de las picaduras generadas por la corrosión. Mientras que la viga V100 rompió a los a 160 días, momento en el que se dio por 
Revista ALCONPAT, Volumen 6, Número 2, Mayo - Agosto 2016, Páginas 129 - 144

finalizado los ensayos. Esto indica que el proceso corrosivo como es sabido, afecta la vida residual del elemento estructural afectado, pero que en particular, las picaduras, pueden disminuirla aún más.

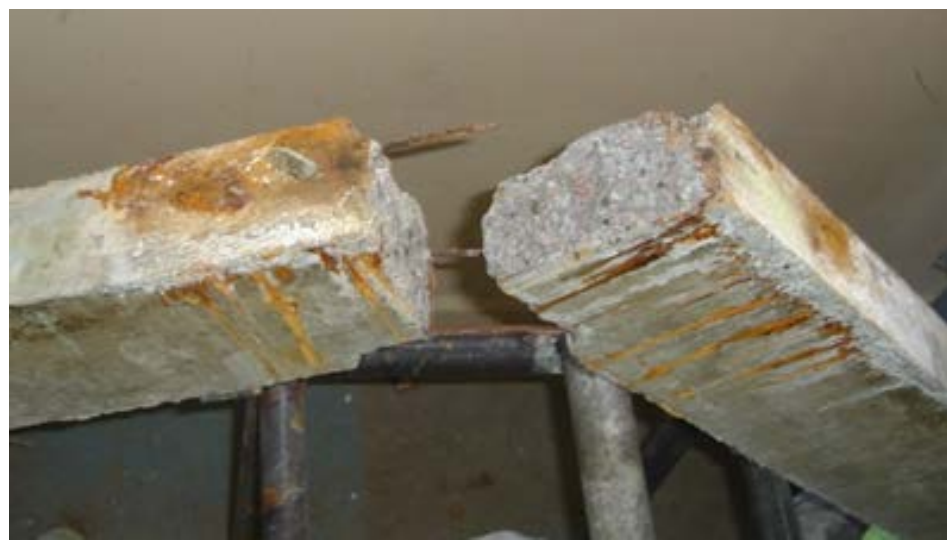

Figura 4. Viga V96 luego del colapso.

El comportamiento electroquímico analizado en las cuatro vigas, a través del seguimiento de sus Potenciales de Corrosión, mostró que en el transcurrir de los primeros 5 días, desde que se conectó el proceso de corrosión acelerada, se llegó al valor mínimo de potencial, donde las vigas con menor recubrimiento (V96 y V100) fueron las que completaron primero su período de activación (con potenciales mínimos entre -478 y $-531 \mathrm{mV}$ ). Posteriormente, se aprecia un aumento de estos potenciales que se lo puede considerar como un proceso de pseudo-pasivación, que es acompañado por la generalización de la fisuración (ver Figura 5), que fue similar en todas las vigas, aunque los potenciales de las dos vigas con menores relaciones recubrimiento / diámetro de armaduras son algo superiores a las otras dos, por lo que se puede decir que están más pasivadas, debido a que tienen una mayor facilidad de ingreso de oxígeno desde el exterior. Este proceso de mencionado, también se encontró en trabajos anteriores realizados en este laboratorio (Aveldaño, 2011; Aveldaño, 2013; Schierloh, 2001).

\subsection{Seguimiento de las Áreas de Fisuración}

Es sabido, que en estructuras de hormigón armado sometidas a flexión, cuando las tensiones, en la zona de tracción, superan la resistencia del hormigón, se generan fisuras, que son denominadas “fisuras por flexión”, identificables por su dirección transversal a la armadura principal de la viga. Esto es inevitable, existan o no problemas de corrosión. Si además, las armaduras están en proceso de corrosión el cuadro de fisuración se ve agravado, por cuanto se superponen ambos efectos: fisuras por flexión y por corrosión.

Debido a que los productos de corrosión poseen un volumen mayor que el material del que se originaron, generan presiones internas que hacen que parte de estos productos rellenen los poros del hormigón y otros salgan al exterior por las fisuras por flexión (si existen). A medida que avanza la corrosión y con la saturación de los poros, los óxidos producen tensiones que, al superar las tensiones de rotura por tracción del hormigón, éste se fisura en dirección longitudinal. Éstas son las que se denominan "fisuras por corrosión". Es necesario aclarar que la división entre fisuras por flexión y por corrosión no es un concepto de muy clara definición, por cuanto, en la medida que evoluciona el proceso de corrosión el progreso de ambas se halla interrelacionado, pudiendo potenciarse mutuamente, como se aclara en el párrafo siguiente. La mencionada diferencia en la dirección de las fisuras, es lo que permite clasificarlas de esta manera.

La presencia de fisuras por flexión (cuando la carga es de magnitud suficiente como para generarlas), hace que se acelere la penetración de oxígeno, agua y cloruros hacia las armaduras, 
Revista ALCONPAT, Volumen 6, Número 2, Mayo - Agosto 2016, Páginas 129 - 144

favoreciendo así el proceso de corrosión de las mismas (ayudando al aumento de las áreas de fisuras por corrosión). Por otra parte, al disminuir la sección de las barras debido a la corrosión, el equilibrio interno de la sección hace que las fisuras por flexión aumenten. Además, al ser las fisuras por flexión vías de egreso al exterior, de una parte de los productos de corrosión, pueden llegar a crecer en espesor y longitud lo que podría incrementar aún más las áreas de fisuración por flexión. Es decir, el crecimiento total de la fisuración implica la disminución de la sección resistente de la viga (por la merma de sección de la armaduras y de la sección útil mecánicamente del hormigón), tal que, en caso de presentarse cargas cercanas a las de rotura del elemento, puede llegar la estructura al colapso, como ocurrió con la viga V96 a los 140 días del ensayo.

Desde que se aplicaron las cargas externas, se comenzaron a medir las fisuras. En la Figura 5 se muestra la evolución de las Áreas de Fisuración Totales en cada viga; es decir, las obtenidas mediante la suma de las áreas de fisuración por flexión, más las de fisuración por corrosión (de igual dirección que la armadura principal de la viga). Cabe recordar, que se denomina Área de Fisuración a la sumatoria de los productos de la longitud por el ancho de cada fisura.

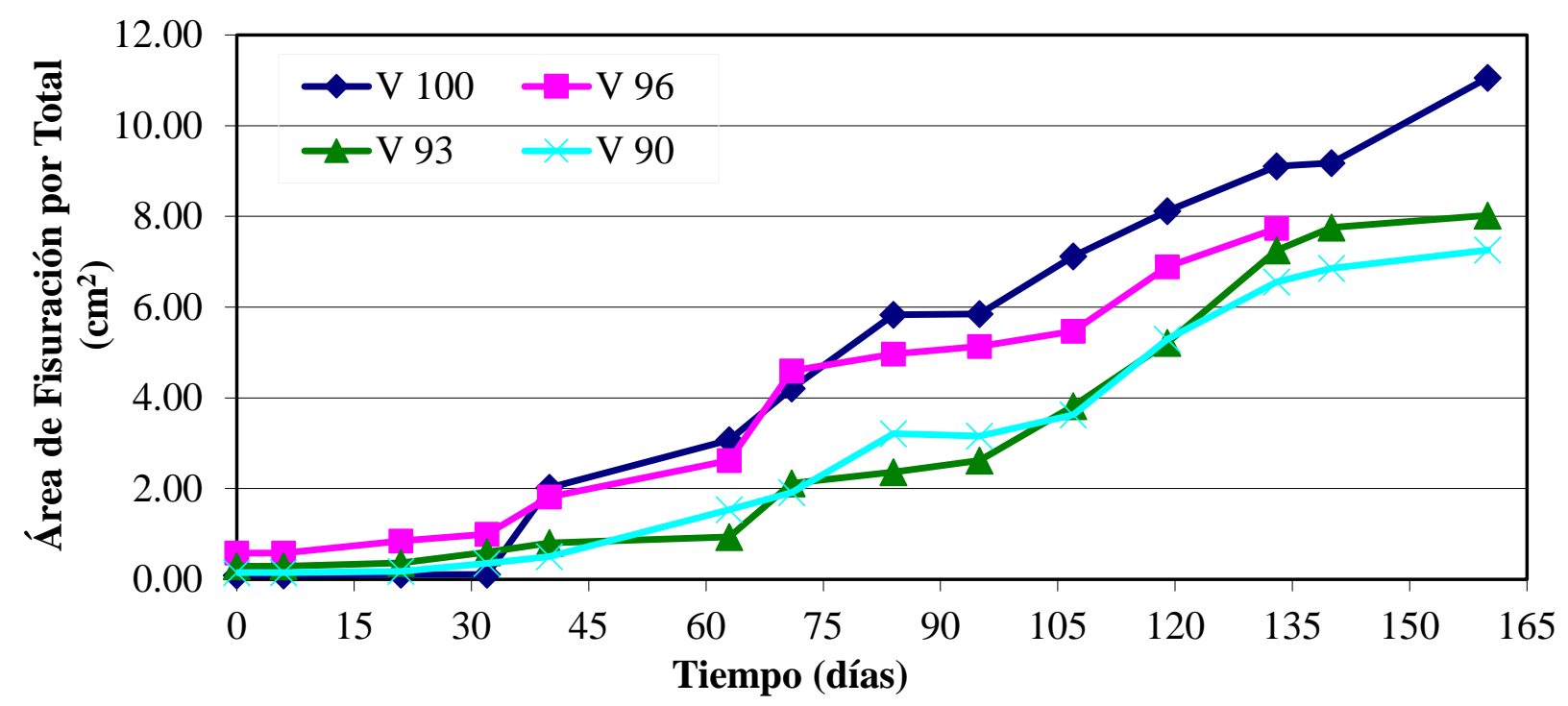

Figura 5. Área de Fisuración Total, en función del Tiempo.

Como era previsible, el inicio de la fisuración por corrosión se manifestó más tarde en las vigas con mayor recubrimiento. Las vigas V90 y V93, tuvieron una evolución del Área de Fisuración Total similar durante el tiempo del ensayo, teniendo en cuenta el grado de dispersión que habitualmente aparece en la mayoría de los trabajos experimentales; mientras que las vigas V96 y V100, mostraron un comportamiento diferenciado, con Áreas de Fisuración Total superiores a las restantes vigas, esto se debe a que poseen una menor relación $r / \phi$ de las armaduras. Sin embargo, si se analiza por separado el Área de Fisuración por Flexión (Figura 6), se observa que la viga V96 presentó valores muy superiores al resto; esto se aprecia claramente con el estado al que llegó dicha viga, donde el deterioro por corrosión se dio en mayor medida en la zona de las fisuras por flexión, mientras que el resto de las vigas, presentaron una fisuración más generalizada. Evidentemente, esta mayor fisuración inicial debida a la flexión, fue una importante vía de ingreso de los cloruros que llegaron hasta las armaduras, presentando la viga V96 las mayores Áreas de Fisuración Total (Figura 5), durante los primeros 30 días del ensayo, luego su comportamiento estuvo dentro de lo esperado. Este comportamiento observado en la viga V96, explica su colapso previo al de la viga V100, y pone en evidencia, por un lado, la influencia de la menor relación r/ $\phi$ de la viga V100, la cual, 
Revista ALCONPAT, Volumen 6, Número 2, Mayo - Agosto 2016, Páginas 129 - 144

teniendo la menor fisuración por flexión durante casi todo el ensayo, presentó una fisuración por corrosión superior al resto; y por otro lado, la importancia que tienen las fisuras por flexión en el deterioro de una estructura sometida a corrosión, como es el caso de la viga V96.

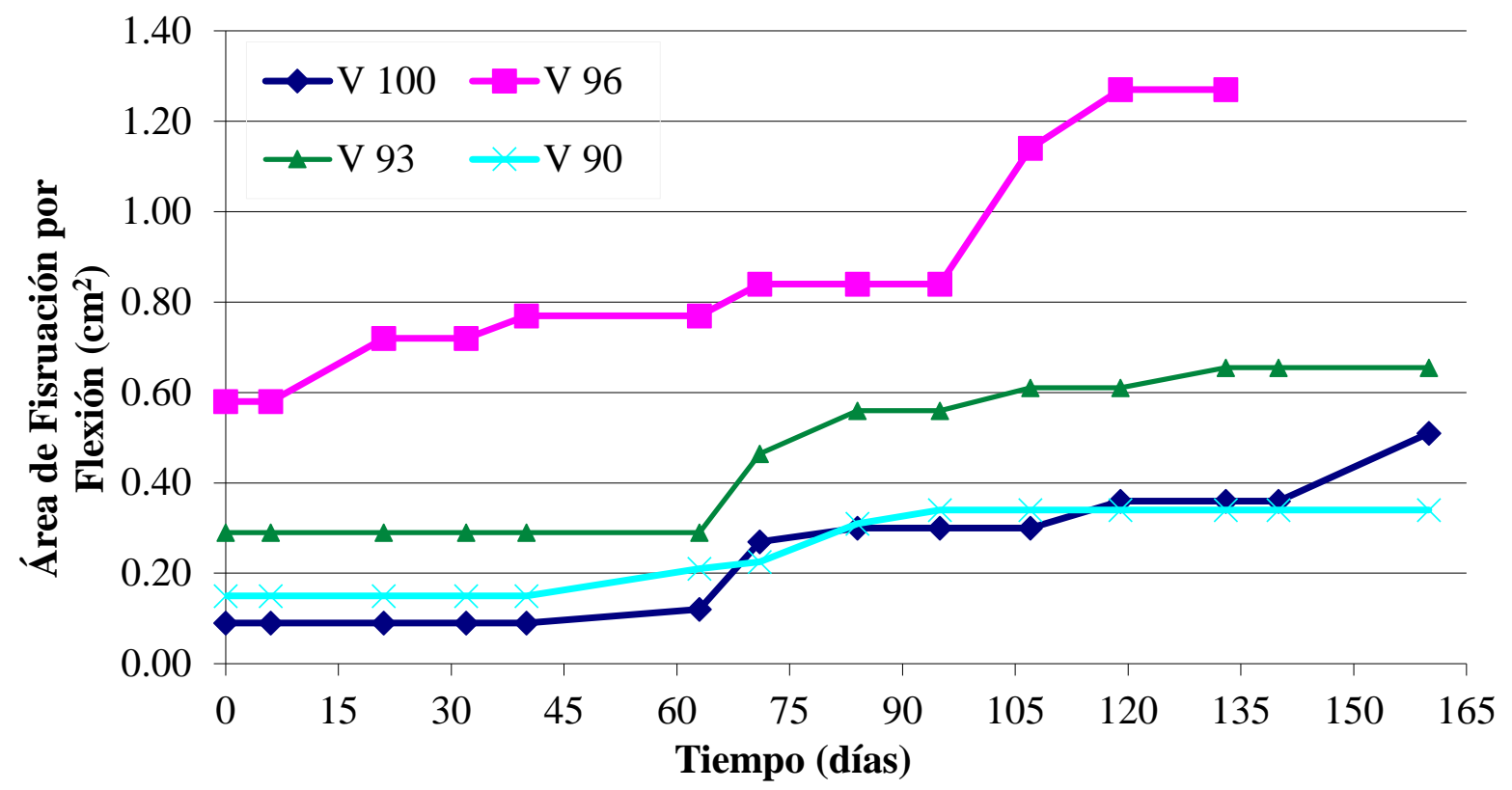

Figura 6. Área de Fisuración por Flexión, en función del Tiempo.

Si bien en este trabajo se analiza el comportamiento frente a un proceso de corrosión de vigas con diferente relación $\mathrm{r}$ / $\phi$ de sus armaduras, las cuales están sometidas a la misma tensión, generada por cargas externas, se puede hacer una comparación de la evolución de la fisuración del recubrimiento observado, con el obtenido en otros trabajos realizados por el mismo grupo de investigación (Moro, 2012), en vigas hechas con hormigones de calidades similares a las de este trabajo, elaborados con o sin agregados reciclados de hormigón y sometidas al mismo proceso corrosivo, pero sin la aplicación de cargas externas. En este caso la viga patrón (sin agregado reciclado), cuenta con una relación $\mathrm{r} / \phi$ de 2,4 y por lo tanto es comparable con la viga V100 del presente trabajo, y al realizar un Análisis de Regresión de las Áreas de Fisuración Totales en ambas vigas, se puede observar que la pendiente es superior en V100 ( $\mathrm{y}=0,074 \mathrm{x}-0,9502)$ que en la viga patrón sin cargas externas $(y=0,068 \mathrm{x}-0,7688)$. Esto se debe a que la presencia de esfuerzos de tracción en V100 en las barras, generan fisuras por flexión, que facilitan el ingreso agentes agresivos del exterior, provocando una aceleración del proceso de corrosión.

En la Figura 7 se aprecia que la aparición de las primeras fisuras visibles por corrosión se demora (la Profundidad media de ataque es proporcional al tiempo, expresión (1)), en la medida que aumenta el espesor del recubrimiento (mayor relación $r / \phi$ ). El incremento del espesor del recubrimiento hace que, se retrase la aparición de las primeras fisuras por corrosión (Figura 7) y su evolución sea más lenta (ver Figura 5) debido a que los elementos agresivos del exterior (cloruros, oxígeno y agua) tardan más en llegar hasta las armaduras. Esta situación, en el caso de armaduras traccionadas con menores relaciones $\mathrm{r} / \phi$, es preocupante, debido a que esa mayor profundidad de ataque en las barras, con menores síntomas externos (fisuración), encierra un riesgo importante, porque se disminuye el tiempo de aviso ante un colapso estructural, siendo más preocupante aún, si hay cloruros que generen picaduras. En la Figura 7, se incorporó además un punto correspondiente a la viga patrón (sin cargas externas y relación $r / \phi=2,4$ ) del trabajo anteriormente mencionado 
Revista ALCONPAT, Volumen 6, Número 2, Mayo - Agosto 2016, Páginas 129 - 144

(Moro, 2012), donde se aprecia que es necesaria una mayor profundidad de ataque para la aparición de las primeras fisuras visibles.

La ecuación obtenida en la Figura 7 mediante un Análisis de Regresión ( $\mathrm{y}=\mathrm{a}+\mathrm{b}$ x), puede compararse con la obtenida en otras investigaciones (Alonso, 1998) donde se utilizaron hormigones elaborados con relaciones agua / cemento entre 0,52 y 0,65, mientras que el resto de las condiciones del ensayo son similares, entre las que se destacan: un rango de variación de la relación recubrimiento / diámetro un poco más amplio que el adoptado en esta publicación, en ambos trabajos se colocaron estribos y se empleó la misma intensidad de corriente. Los resultados logrados en el trabajo citado, fueron: $\mathrm{a}=7,53 \mathrm{y} \mathrm{b}=9,32$, con $\mathrm{R}^{2}=0,92$. La pendiente de esta recta es muy inferior a la obtenida en el presente trabajo $(\mathrm{a}=13,71)$, por lo tanto, se puede decir que, en las estructuras sometidas a cargas externas, se acelera la degradación generada por la corrosión de sus armaduras, aún para hormigones elaborados con una relación agua / cemento algo inferior a las de la referencia.

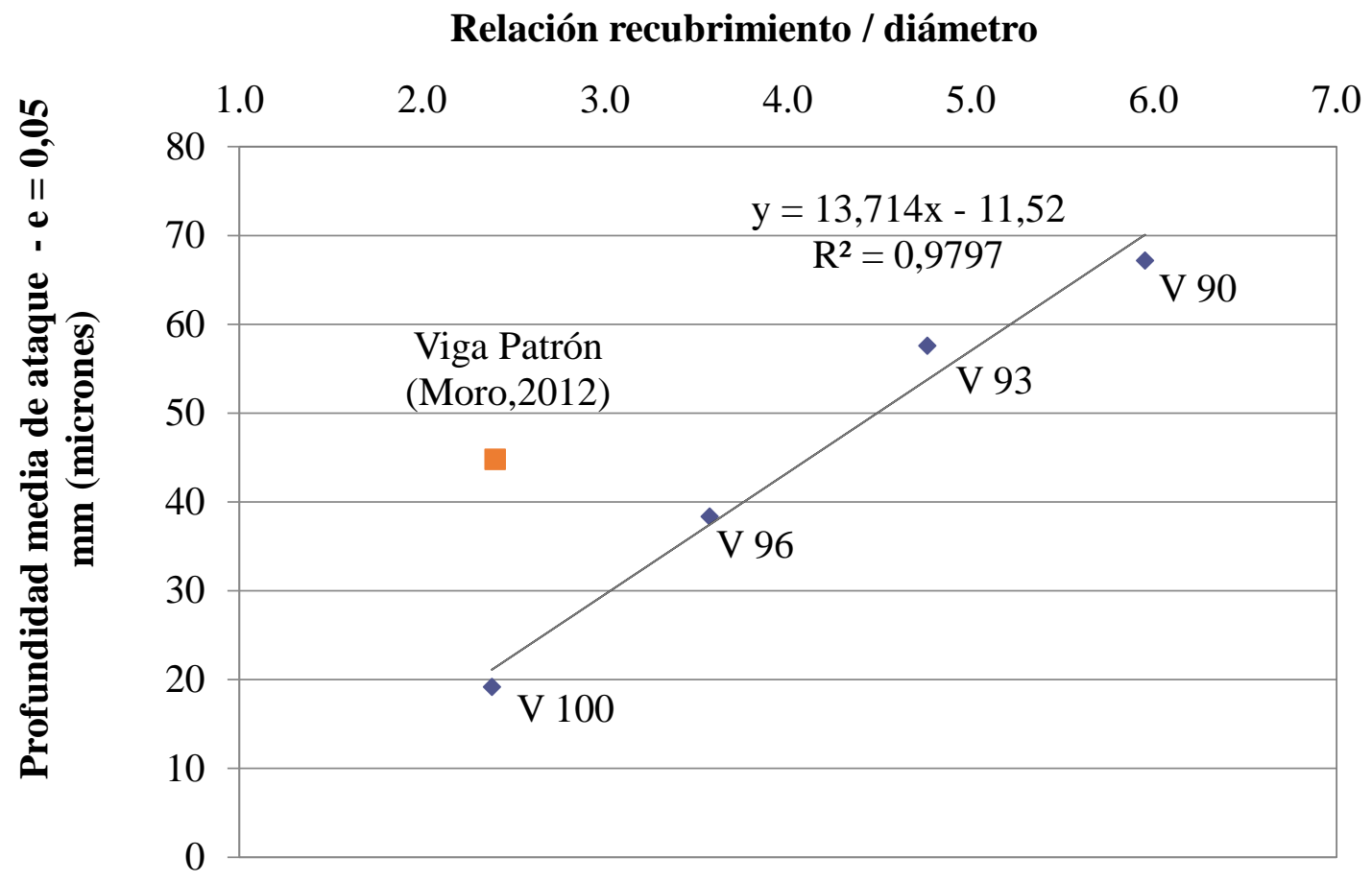

Figura 7. Relación entre la profundidad media de ataque a las armaduras que produjo la primer fisura visible (e = 0,05 mm) por corrosión y la Relación Recubrimiento / Diámetro de la barra.

Una vez finalizado el ensayo, se descubrieron las armaduras, retirando sus recubrimientos. En la inspección visual de las mismas, pudo observarse que, debido a la acción de los cloruros (a pesar de la baja concentración adoptada), la corrosión sobre las barras en general no fue uniforme, sino que se observaron algunas zonas de corrosión localizada (picaduras), sin mostrar ningún patrón en particular. Sin embargo, en algunos casos se encontraron mayores picaduras en las zonas cercanas a las fisuras por flexión, como ocurrió en la Viga V96, donde la sección de colapso, coincidió con una fisura por flexión.

\subsection{Seguimiento de los Anchos Máximos de Fisuras}

En la Figura 8, se muestra la variación del Ancho Máximo de Fisuración, en función del Tiempo. Se puede observar, que el Ancho Máximo de Fisuración, durante la mayor parte del ensayo, fue superior en la viga V96, por la influencia de la fisuración por flexión. En la evolución del resto de las vigas no se apreciaron grandes diferencias, debido a que la distribución de la fisuración se 
Revista ALCONPAT, Volumen 6, Número 2, Mayo - Agosto 2016, Páginas 129 - 144

desarrolló diferente en cada viga, ya sea en forma más generalizada o más localizada, sin generar diferencias evidentes entre las distintas muestras ensayadas.

Si se compara la evolución de los Anchos Máximos de Fisuración con la de las Áreas de Fisuración (Figura 5), se puede ver que estas últimas son un indicador más representativo de la evolución general del deterioro, debido a que el Ancho Máximo es habitual que varíe de ubicación en la viga, durante el proceso de corrosión; además, suele evolucionar a saltos, en función de la aparición de nuevas fisuras que hacen que se redistribuyan las tensiones internas en el recubrimiento. Por ejemplo, en Figura 8 se aprecia, que la viga V90 es la que tiene mayor fisuración, entre los 40 y 90 días, cuando en realidad tendría que ser la que presentase los menores valores. Esta situación también se la encontró en otros trabajos (Aveldaño, 2009, Aveldaño, 2013). No obstante, se incluye esta figura debido a que en las normativas es común que se introduzcan limitaciones del ancho máximo de fisuras, por ello es usado como referencia en mucha bibliografía internacional. Cabe acotar que, habría que hacer investigaciones para normalizar el Área de Fisuración, para poder realizar comparaciones valederas entre distintas vigas deterioradas, por ejemplo, en función de la longitud del elemento afectado o del largo del área corroída, para luego establecer indicadores representativos del deterioro y limitarlo.

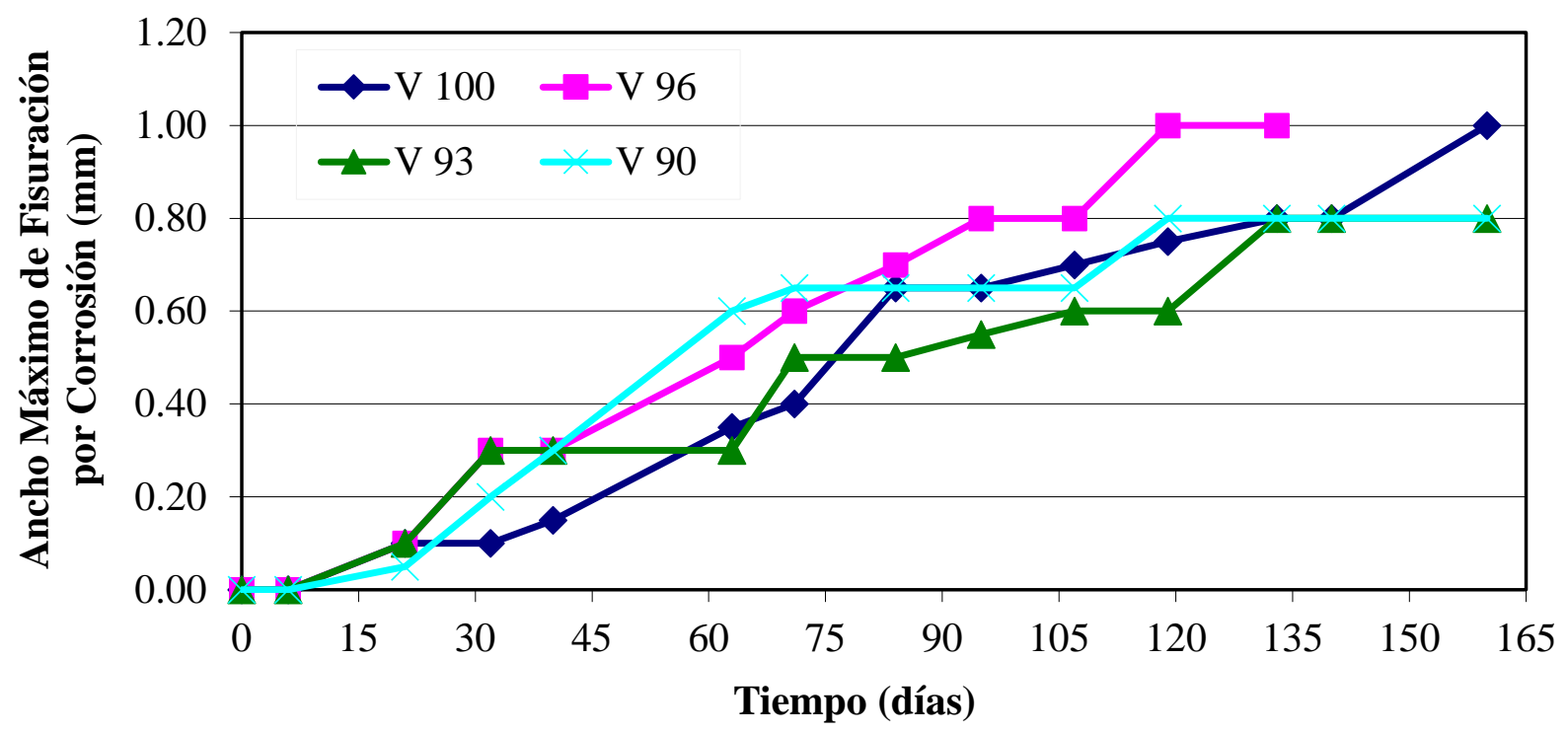

Figura 8. Ancho Máximo de Fisuración, en función del Tiempo.

\section{CONCLUSIONES}

En este trabajo experimental, se encontró que el deterioro por corrosión de sus armaduras, de vigas expuestas a cargas externas fue mayor que si no estuviesen cargadas y que el espesor del recubrimiento tiene influencia en este proceso, todo lo cual afecta la vida residual y la capacidad portante de la estructura afectada. En particular, se comprobó que:

- En vigas cargadas, con armaduras traccionadas afectadas por corrosión acelerada, al incrementarse la relación recubrimiento de hormigón / diámetro de las armaduras, lleva más tiempo en aparecer las primeras fisuras por corrosión y su evolución se hace más lenta, poniéndose en evidencia en las Áreas Totales de Fisuración. El efecto de protección generado por el mayor recubrimiento, hace que los elementos agresivos provenientes del exterior (cloruros, oxígeno, agua) requieran más tiempo para llegar hasta las armaduras, aminorando la velocidad del proceso; 
Revista ALCONPAT, Volumen 6, Número 2, Mayo - Agosto 2016, Páginas 129 - 144

- Las fisuras del recubrimiento de hormigón, originadas por la flexión producida por cargas externas, favorecen el incremento de la fisuración por corrosión de las armaduras, aumentando la fisuración total y por consiguiente, influyendo en la degradación posterior de la estructura.

Desde el punto de vista práctico, las conclusiones anteriores permiten determinar, que en construcciones de hormigón armado cargadas y ubicadas en ambientes agresivos, conviene disminuir todo lo posible la fisuración por flexión, originada por las cargas de servicio, aumentando asimismo la relación recubrimiento / diámetro de las armaduras, para disminuir los efectos de corrosión en las mismas.

\section{AGRADECIMIENTOS}

Los autores expresan su agradecimiento a la Dra. Carla Priano, Ing. Lilia Señas y a los técnicos J.P. Gorordo del Laboratorio de Modelos Estructurales y D. Smith del Laboratorio de Estudio y Ensayos de Materiales, U.N.S., por su colaboración en la elaboración de los ensayos. Además, se agradece a la Secretaría General de Ciencia y Tecnología y al departamento de Ingeniería de la Universidad Nacional del Sur por su aporte económico e institucional para la realización de estas investigaciones.

\section{REFERENCIAS}

Almusallam, A. A., Al-Gahtani, A. S., Aziz, A. R. (1996), "Effect of reinforcement corrosion on bond strength", Construction and Building Materials, V. 10, No. 2, pp. 123-129.

Alonso, M. C., Andrade, C., Rodríguez, J., Diez, J. M. (1998), “Factors controlling cracking in concrete affected by reinforcement corrosion”, Materials and Structures, Nr. 31, pp. 435-445.

Alonso, M. C., Andrade, M. C., Rodríguez, J., Casal, J., García, M. (1994), "Evaluación experimental de la fisuración del hormigón producida por la corrosión de las armaduras”, Hormigón y Acero, No. 194, pp. 29-42.

Al-Sulaimani, J., Kaleemullah, M., Basunbul, I. A., Rasheeduzafar (1990), “Influence on corrosion and cracking on bond behaviour and strength of reinforced concrete members", ACI Structural Journal, V. 87, Nr 2, pp. 220-231.

American Society for Testing and Materials: ASTM C876, "Standard test method for half cell potential of reinforcing steel in concrete”, Philadelphia, U.S.A, (1980).

Andrade, M. C., Alonso, M. C., Molina, F. J. (1993), “Cover cracking as a function of bar corrosion: Part I - Experimental test”, Materials and Structures, Nr. 26, pp. 453-464.

Aveldaño, R. R., Ortega, N. F. (2009), "Influence of reinforcement distribution in the corrosive process of reinforced concrete beams", Magazine of Concrete Research, Thomas Telford, V. 61, Nr. 3, pp. 213-220.

Aveldaño, R. R., Ortega, N. F. (2011), “Characterization of Concrete Cracking due to Corrosion of Reinforcements in Different Environments”, Construction and Building Materials, V.25, pp.630-37. Aveldaño,R. R., Ortega, N. F.(2013), "Behavior of concrete elements subjected to corrosion in their compressed or tensed reinforcement”, Construction and Building Materials, Nr. 38, pp. 822-828.

Calabrese, L., Campanella, G., Proverbio, E. (2013), “Identification of corrosion mechanisms by univariate and multivariate statistical analysis during long term acoustic emission monitoring on a pre-stressed concrete beam”, Corrosion Science, V. 73, pp. 161-171.

Elfergani, H. A., Rhys, P., Holford, K. M. (2013), “Damage assessment of corrosion in prestressed concrete by acoustic emission”, Construction and Building Materials, V. 40, pp. 925-933. 
Revista ALCONPAT, Volumen 6, Número 2, Mayo - Agosto 2016, Páginas 129 - 144

Fumin, L., Yingshu, Y., Chun-Qing, L. (2011), “Corrosion propagation of prestressing steel strands in concrete subject to chloride attack”, Construction and Building Materials, V. 25, Nr.

10, pp. 3878-3885.

Hariche, L., Ballim, Y., Bouhicha, M. Kenai, S. (2012), “Effects of reinforcement configuration and sustained load on the behaviour of reinforced concrete beams affected by reinforcing steel corrosion”, Cement and Concrete Composites, V. 34, Nr. 10, pp. 1202-1209.

Instituto Argentino de Normalización y Certificación IRAM 1627: “Agregados. Granulometría de los agregados para hormigón”, Buenos Aires, (1997).

Instituto Argentino de Racionalización de Materiales - Instituto Argentino de Siderurgia, Norma U 500-528: "Barras de acero conformadas de dureza natural, para armadura en estructuras de hormigón”, Buenos Aires, (1989).

Instituto Argentino de Normalización y Certificación IRAM 1871: "Método de ensayo para determinar la capacidad y la velocidad de succión capilar de agua del hormigón endurecido", Buenos Aires, (2005).

Instituto Argentino de Normalización y Certificación IRAM 1546: "Hormigón de cemento portland. Método de ensayo de compresión”, Buenos Aires, (1992).

Instituto Argentino de Normalización y Certificación IRAM 1658: Hormigón. "Método de ensayo de tracción simple por compresión diametral”, Buenos Aires, (1995).

Instituto Argentino de Normalización y Certificación IRAM 1534: “Hormigón de cemento portland. Preparación y curado de probetas para ensayos en laboratorio”, Buenos Aires, (1985).

Malumbela, G., Moyo, P., Alexander, M., (2009), “Behaviour of RC beams under sustained service loads”, Construction and Building Materials, V. 23, Nr. 11, pp. 3346-3351.

Melchers, R. (2001), “Assessment of existing structures, approaches and research needs”, Journal of Structural Engineering, ASCE, pp. 406-411.

Moro, J. M., Meneses, R. S., Ortega, N. F., Aveldaño, R.R., Señas, L., Priano C. V. (2012), "Corrosión de Armaduras en Estructuras de Hormigón Reciclado con Tratamientos Previos", CINPAR 2012, VIII Congreso Internacional sobre Patología y Recuperación de Estructuras, 11 páginas.

Ortega, N. F., Alonso, M. C., Andrade, M. C., López, C. (2001), “Análisis de la fisuración ocasionada por la corrosión de las armaduras activas de elementos pretensados”, Coloquia, Madrid.

Peralta, M. H., Rivas, I. E., Ortega, N. F. (2006), “Análisis Numérico de la Fisuración Superficial de Estructuras de Hormigón Armado por Efecto de la Corrosión”, Informes de la Construcción, V. 58, No. 501, pp. 51-58.

Rodríguez, J., Ortega, L. M., Casal, J., Vidal, M. A. (1993), “Disminución de la adherencia entre hormigón y barras corrugadas debido a la corrosión”, Hormigón y Acero, No 189, pp. 49-65.

Rodríguez, J., Ortega, L. M., García, A. M. (1993), “Medida de la velocidad de corrosión de las armaduras en estructuras de hormigón, mediante un equipo desarrollado dentro del proyecto Eureka EU 401”, Hormigón y Acero, No. 189, pp. 79-91.

Rodríguez, J., Ortega Basagoiti, L. M., Casal, J., Diez, J. M. (1996), “Comportamiento estructural de vigas de hormigón con armaduras corroídas”, Hormigón y Acero, No. 200, pp.113-131.

Rodríguez, J., Ortega Basagoiti, L. M., Casal, J., Diez, J. M. (1998), “La corrosión de armaduras y la vida residual de las estructuras de hormigón”, Hormigón y Acero, No. 208, pp. 63-78.

Influencia del espesor del recubrimiento de elementos de hormigón armado expuestos a... 
Revista ALCONPAT, Volumen 6, Número 2, Mayo - Agosto 2016, Páginas 129 - 144

Schierloh M. I. (2003)., “Corrosión de armaduras. Características que debe tener el hormigón para aumentar la protección”, Tesis de Magíster en Ingeniería, Departamento de Ingeniería, Universidad Nacional del Sur, Director de Tesis: Ortega N.F.

Schierloh M. I., Ortega N. F., Señas L. N. (2001), "Relación entre Algunas Propiedades del Hormigón del Recubrimiento y el Proceso Corrosivo de las Armaduras”, $14^{\circ}$ Reunión de Asociación Argentina de Tecnología del Hormigón, pp. 125-132.

Torres-Acosta, A. A., Navarro-Gutierrez, S., Terán-Guillén, J. (2007), “Residual flexure capacity of corroded reinforced concrete beams”, Engineering Structures, V. 29, Nr. 6, pp. 1145-1152.

Tuutti K. (1982); “Corrosion of steel in concrete”, Swedish Cement and Concrete Institute (CIB) pp. 4-82, Stockholm, Sweden

Yu, L., François, R., Dang, V. H., L'Hostis, V., Gagné, R., “Development of chloride-induced corrosion in pre-cracked RC beams under sustained loading: Effect of load-induced cracks, concrete cover, and exposure conditions”, Cement \& Concrete Research, Nº67, 2015, pp.246-258. 